\title{
VUV HIGH RESOLUTION ABSORPTION SPECTRA OBTAINED WITH SYNCHROTRON LIGHT, AND INTERPRETATIONS
}

\author{
A.M. Baig, J.P. Connerade, W.R.S. Garton, J. Hormes, \\ C. Mayhew, G. Noldeke and K. Sommer.
}

Physikalisches Inttitut, der Universitat Bonn \& Blackett Laboratory Imperial College, London

For over ten years an expanding collaborative programme of experimental work on the absorption spectra of neutral atomic and simpler molecular gases and vapours, and on similar species "matrix-isolated" in rare-gase solids, has been in progress. The experimental work has centered on use of background continua from the 0.5 and $2.6 \mathrm{GeV}$ synchrotrons in Bonn, - high resolution work being based mainly on the smaller machine. The instrumentation includes most significantly for the work reported here - a 3-metre Eagle-style vacuum spectrograph equipped with "holographic" gratings of 5,000 $6,0001 / \mathrm{mm}$ spacing, and usable over the range $200-2,000 \AA$.

As regards absorption spectroscopy of neutral atoms in the VUV, the objective is to extend understanding of the atomic mechanics involved in inner-shell photoexcitation. The work in Bonn has produced a very large amount of new information on inner-shell and double- or multiple-excitations, of about half of the periodic system up to Bi $I$. This work has stimulated attempts at better theoretical

interpretations, which have now become elaborate. A few cases in Figs. 1-4 11lustrate the performance of the "holographic $3 \mathrm{~m}$ ".

1. $\mathrm{Ba} I$ - From earlier work 14 series attaching to 12 limits were identified by 1979. Most recently the spectrum was re-examined with the $3 m$ instrument, giving a dispersion increase by a factor of 15 (see Baig et al., 1984 for interim report). Spectra were obtained in second order $(0.27 \mathrm{~A} / \mathrm{mm})$, by using a column of $\mathrm{Hg}$ vapour to suppress the first order of the background continuum in the range below $1178 \AA$. In adition to improving and extending previous classification two further unexpected limits have been identifled. Fig. I shows a portion of spectrum. 2. Rare Gases - Figure 2 shows the spectrum of Ne I, in first order. Second-order spectra have also been obtained by use of the Hg I order-sorter mentioned above. This spectrum has previously been studled by others, but the Bonn instrument has given improved resolution by a factor of about 10 . Though He $I$ is generally recognized as being the exception among the rare gases, $\mathrm{Ne} I$ is more subtly unique in not being followed by growth of a d-subshell. This fact makes the Ne I autoionization structure between the levels of the ground doublet of the ion quite different from that in the cases of the heavier inert gases. Thus, as can be seen in Fig. 2, autoionization widths are small and in contrast to $\mathrm{A}, \mathrm{Kr}$, Xe, - the ns transitions are broader than the nd. 3. Yb I - This spectrum has been the subject of a number of papers over the last few years. Figure 3 shows a portion of a higher resolution plate near $1400 \AA$, from which recognition of a new series due to excitation of a $4 f$ electron mixed with double-electron transitions has followed.

The foregoing are a few examples of work concerned with "internal 
field" effects in atoms, i.e., effects ascribable to interactions/ correlations of the electrons of several shells or subshells. Other important cases (e.g., the alkalis or the $Z \pi I$ - Hg I group) have been recently reported or are in course of interpretations. We are left in this area of work with the challenge of the three rows of transition elements - the important $C, N, 0$, and halogen groups and a few likewise important cases of light elements like Al $\mathrm{I}$. The wide-open field concerns proper quantitative absorption cross-section measurements. Accordingly, replacement of photography by linear-diode arrays ("reticons") is being initiated.

4. Molecular Spectra - The 3-meter spectrograph and the $0.5 \mathrm{GeV}$ machine have been used by one of us (C.M.) for a comprehensive exploration of the dihydrides and deutrides of the oxygen group (i.e., $\mathrm{H}_{2} \mathrm{O}$ to $\mathrm{D} \mathrm{Te}$ ). The spectra have produced a number of new Rydberg series in the range 900-1700\%. A nice example is shown in Fig. 4 where the Rydberg bands of $\mathrm{H}, \mathrm{Te}$ can be followed to $\mathrm{n}=30$. Figure 5 shows the variation of rotational structure of the first member of the so-called E-series of this group of triatomics. 5. Zeeman Patterns - The first objective here has been to extend work on magnetic effects in long Rydberg series and adjacent continua, so far confined to the alkalis and alkaline-earth elements and above $2,000 \AA$, into the VUV. Initial experiments were performed with a solenoid on temporary loan from ANL (Dr. F.S. Tomkins). A larger similar solenoid is just being commissioned in Bonn. However, use of the Argonne solenoid led to an unexpected bonus, in the shape of a promising method for VUV oscillator strength measurement, based essentially on magnetic rotation of plane polarized light (Faraday effect). Figure 6 illustrates.

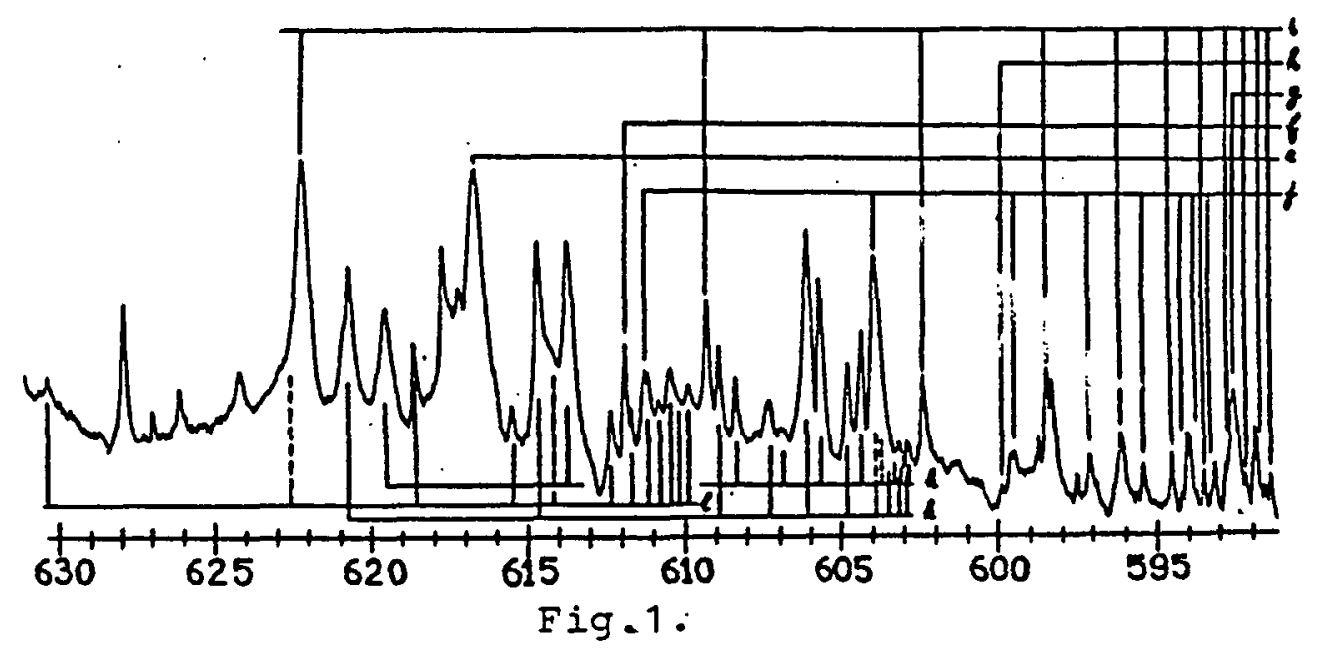



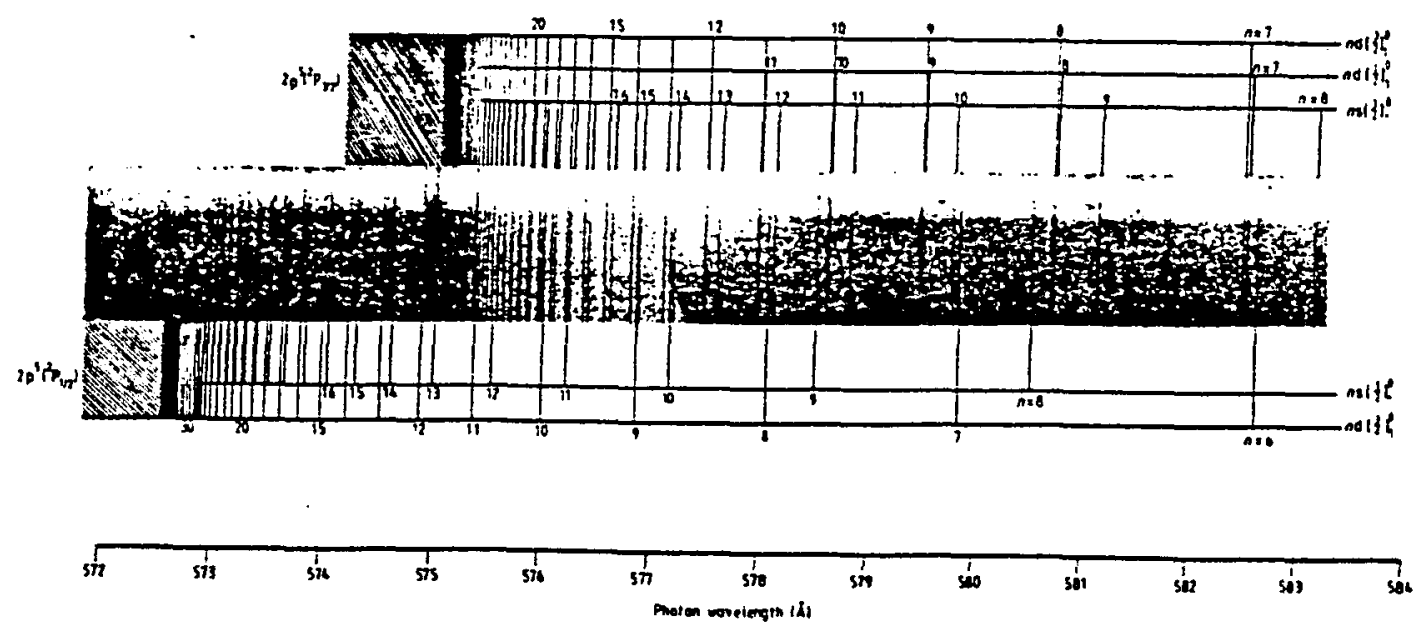

Fig. 2 .

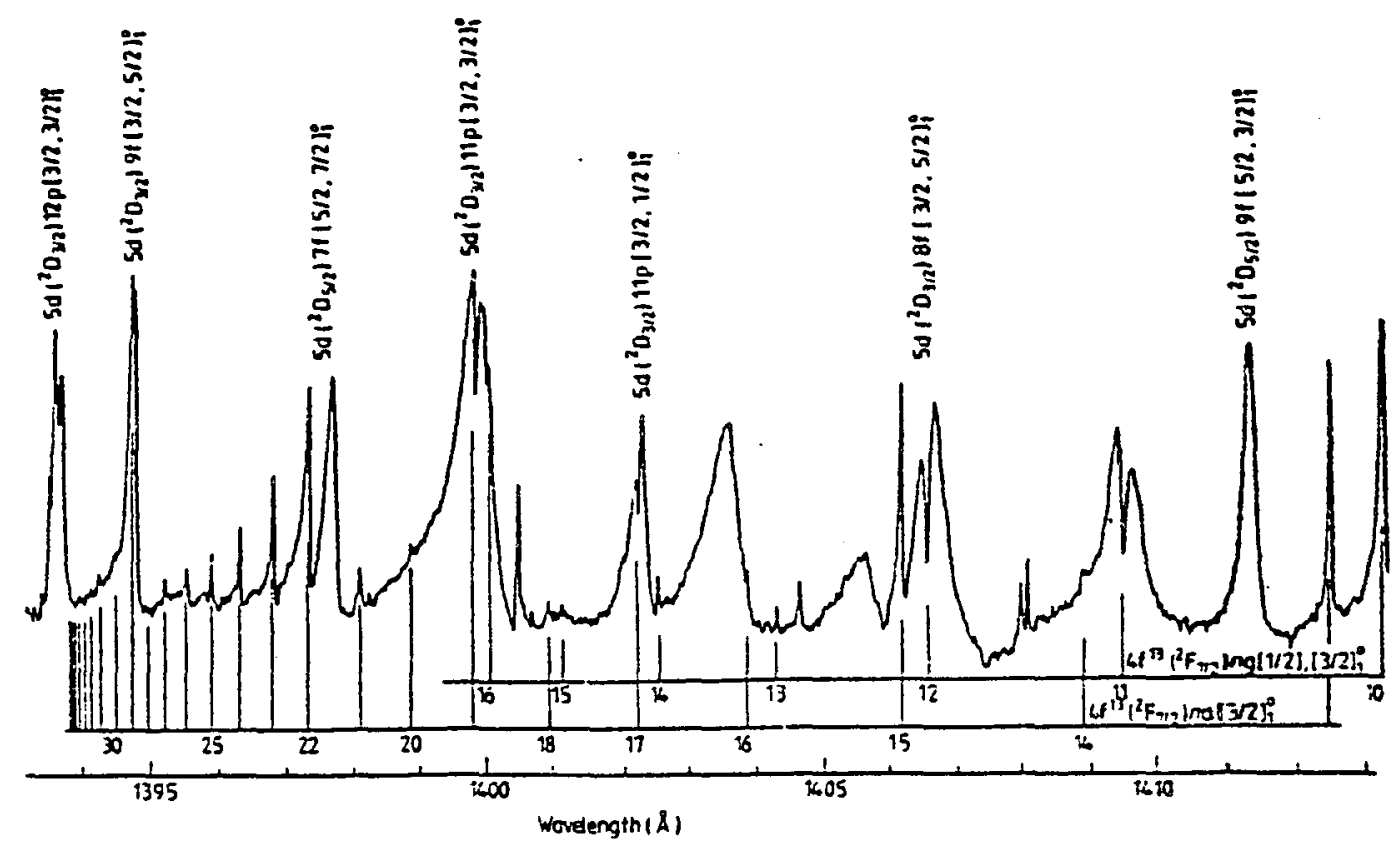

Fig.3.

Densitometer trace of photoabsorption spectrum of $\mathrm{Yb}$ i showing the high members of the series due to single excitation from the df subshell. Note how sharp the lines remain except in the immediate vicinity of the strong perturber.

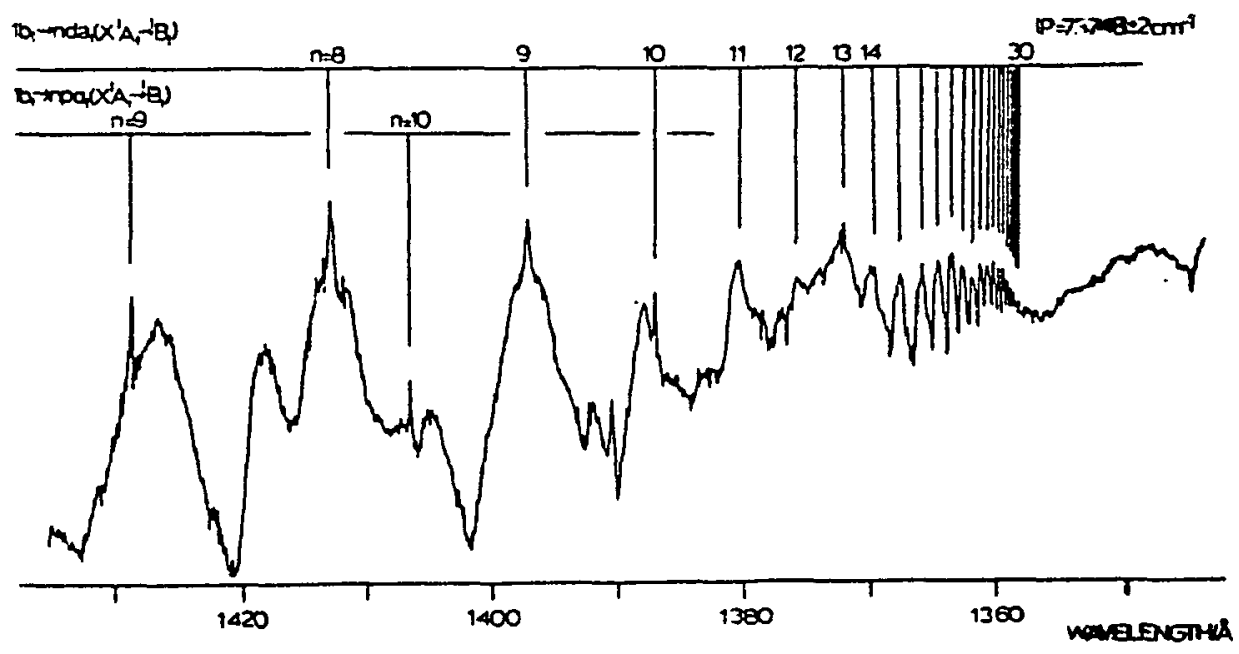

Fig.4. 

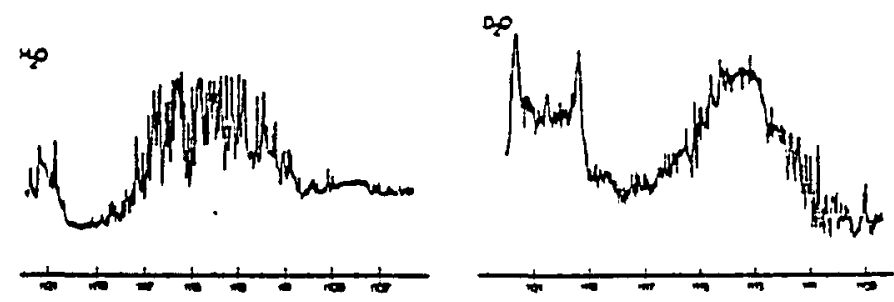

ms
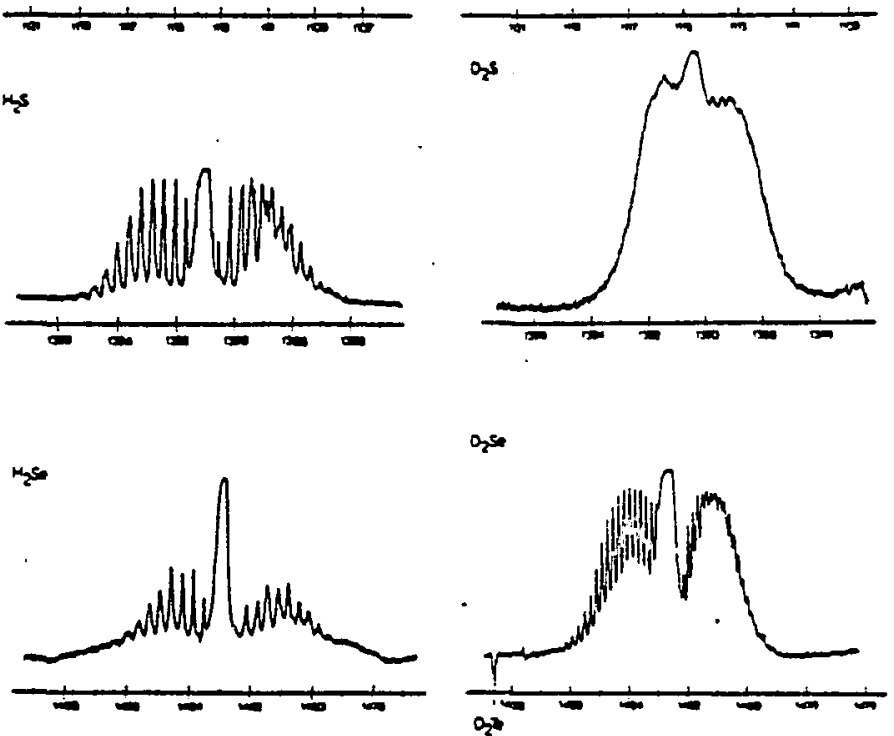

o,se
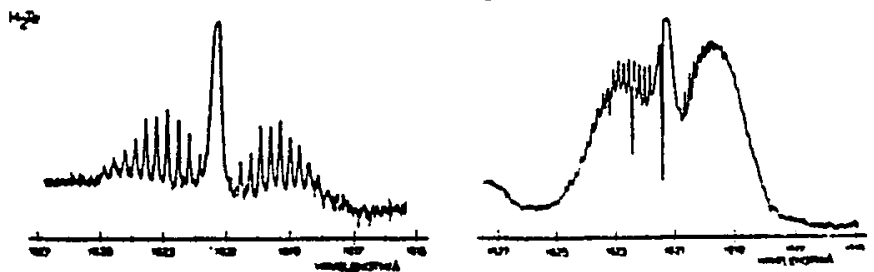

Fig. 5 .

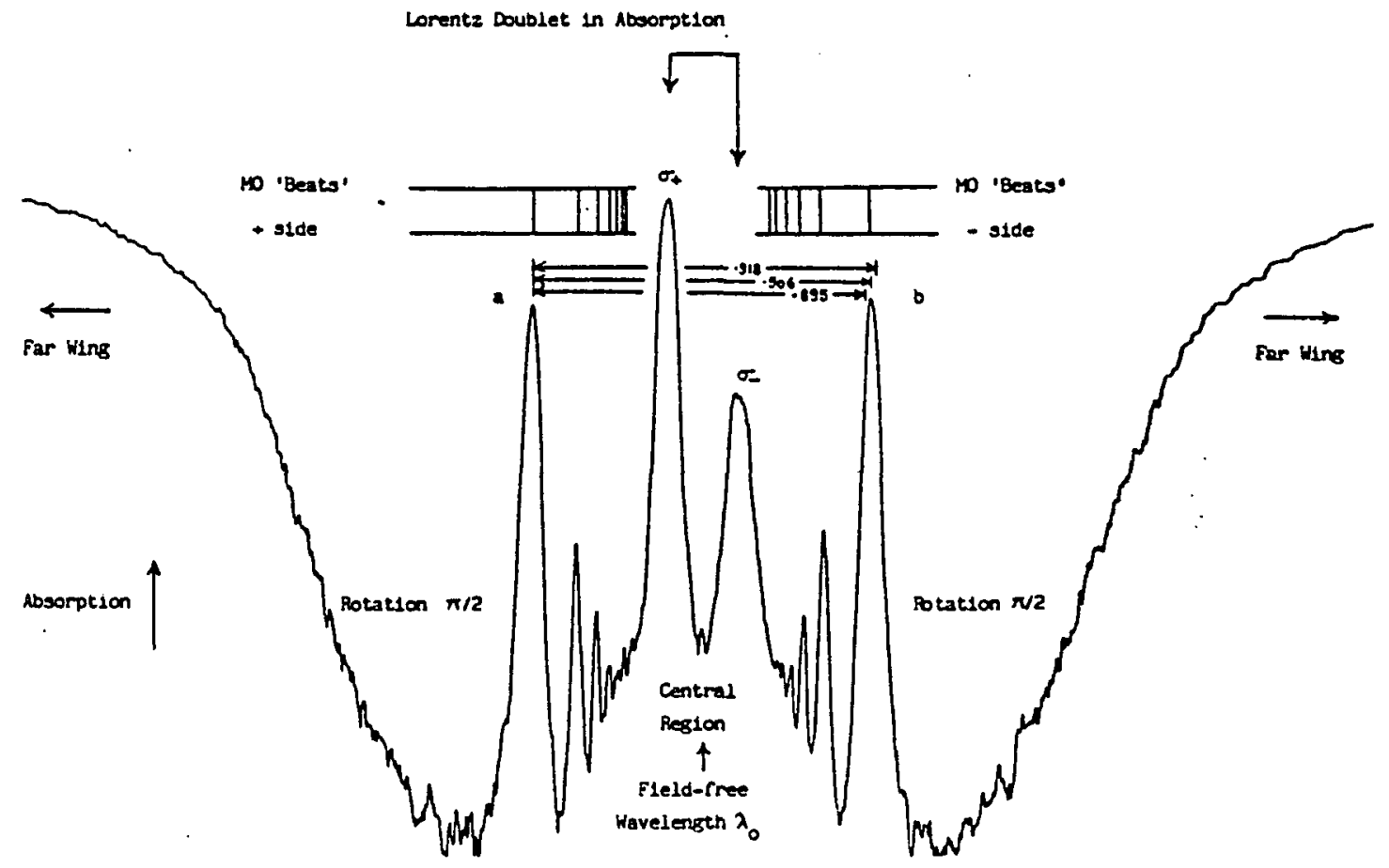

Fig.6. 